\title{
Detection of Chemotherapy-Resistant Pancreatic Cancer Using a Glycan Biomarker
}

\author{
ChongFeng Gao', Luke Wisniewski ${ }^{1}$, Ying Liu ${ }^{1}$, Ben Staal ${ }^{1}$, lan Beddows ${ }^{1}$, Dennis \\ Plenker $^{2}$, Mohammed Aldakkak ${ }^{3}$, Johnathan Hall ${ }^{1}$, Daniel Barnett ${ }^{1}$, Mirna Kheir Gouda ${ }^{2}$, \\ Peter Allen ${ }^{4}$, Richard Drake ${ }^{5}$, Amer Zureikat ${ }^{6}$, Ying Huang ${ }^{7}$, Douglas Evans ${ }^{3}$, Aatur \\ Singhi ${ }^{6}$, Randall E. Brand ${ }^{6}$, David A. Tuveson ${ }^{2}$, Susan Tsai ${ }^{3}$, and Brian B. Haab ${ }^{1}$ \\ ${ }^{1}$ Center for Cancer and Cell Biology, Van Andel Institute, Grand Rapids, MI 49503, USA \\ ${ }^{2}$ Lustgarten Foundation Pancreatic Cancer Research Laboratory, Cold Spring Harbor \\ Laboratory, Cold Spring Harbor, NY 11724, USA \\ ${ }^{3}$ Department of Surgery, Medical College of Wisconsin, Milwaukee, WI 53226, USA \\ ${ }^{4}$ Division of Surgical Oncology, Duke University School of Medicine, Durham, North Carolina \\ 27710, USA \\ ${ }^{5}$ Cell and Molecular Pharmacology and Experimental Therapeutics, Medical University of South \\ Carolina, Charleston, SC 29425, USA \\ ${ }^{6}$ Division of Gastrointestinal Surgical Oncology, University of Pittsburgh Medical Center, \\ Pittsburgh, PA 15213, USA \\ ${ }^{7}$ Vaccine and Infectious Disease Division, Fred Hutchinson Cancer Research Center, Seattle, \\ WA 98109, USA
}

Corresponding Author

Brian B. Haab, PhD

Van Andel Institute

333 Bostwick NE, Grand Rapids, MI 49503

(616) 234-5268

Brian.haab@vai.org

Short Title: Detecting Drug-Resistant PDAC

Conflict of Interest Statement: The authors have declared that no conflict of interest exists.

\section{Grant Support}

U01CA152653, Early Detection Research Network, National Cancer Institute, to R.E.B. and B.B.H.; U01CA226158, Alliance of Glycobiologists for Cancer Detection, National Cancer Institute, B.B.H.; D. A. Tuveson: Distinguished Scholar and Director of the Lustgarten

Foundation-Designated Laboratory of Pancreatic Cancer Research. D. Plenker is supported by the German Research Foundation (DFG) (PL 894/1-1).

\section{Author Contributions}

B.H. and C.G. conceived and designed the experiments. C.G., L.W., Y.L., and B.S. conducted most of the laboratory experiments. J.H. and D.B. assisted in data analysis. I.B. analyzed the RNA-seq data and performed bioinformatic analysis. D.P and D.A.T generated organoids slides and conditional media. H.Y. performed statistical analyses and assisted in data interpretation. M.A. and S.C provided patient serum samples and contribute to data analysis. M.K.G., P.A., R.D., A.Z., D.E., A.S., and R.E.B provided specimen resources and assisted in data interpretation. B.H. wrote the manuscript, and all authors reviewed and commented on the manuscript. 


\section{Detecting Drug-Resistant PDAC}

\section{Abstract}

Background and Aims. A subset of pancreatic ductal adenocarcinomas (PDACs) is highly resistant to systemic chemotherapy, but no markers are available in clinical settings to identify this subset. We hypothesized that chemotherapy-resistant PDACs express a glycan biomarker called sTRA. Methods. We tested this marker to identify treatment-resistant PDAC in multiple systems: sets of cell lines, organoids, and isogenic cell lines; primary tumors; and blood plasma from cohorts of human subjects. Results. Among a panel of 27 cell lines, high levels of cellsurface sTRA identified higher resistance to seven chemotherapeutics used against PDAC. Using primary tumors from two different cohorts, patients who were positive for a geneexpression classifier for STRA received no statistically significant benefit from adjuvant chemotherapy, in contrast to those negative for the signature. In another cohort, using direct measurements of sTRA in tissue microarrays by quantitative immunofluorescence, patients who were high in STRA again had no statistically significant benefit from adjuvant chemotherapy. Further, a blood-plasma test for the sTRA glycan identified the PDACs that showed rapid relapse following neoadjuvant chemotherapy. This blood test performed with $96 \%$ specificity and $56 \%$ sensitivity in a blinded cohort using samples collected before the start of treatment. Conclusion. These findings establish that tissue or plasma sTRA can identify PDACs that are resistant to neoadjuvant or adjuvant chemotherapy. This capability could help apply systemic treatments more precisely and facilitate biomarker-guided trials targeting resistant PDAC.

\section{Keywords}

Pancreatic cancer; molecular subtypes; blood test; glycans; chemoresistance; CA19-9; sTRA 


\section{Detecting Drug-Resistant PDAC}

\section{Introduction}

Systemic therapy is considered necessary for all patients with pancreatic ductal adenocarcinoma (PDAC), even those with localized disease, because most patients already have occult metastases at the time of diagnosis ${ }^{1}$. Chemotherapy particularly benefits patients who have surgical resection of the tumor. In a seminal study that established adjuvant chemotherapy (chemotherapy applied after surgery) as standard of care for PDAC, the median disease-free survival after surgery improved to 13.4 months with gemcitabine from 6.9 months with observation ${ }^{2}$. Further improvements in chemotherapy were demonstrated using the stronger FOLFIRINOX regimen ${ }^{3}$, or gemcitabine in combination with capecitabine ${ }^{4}$ or nabpaclitaxel $^{5}$. Systemic therapy is increasingly applied prior to surgery-called neoadjuvant therapy-in order to increase the percentage of patients who receive chemotherapy ${ }^{6}$, since some patients have a delay or reduction in their adjuvant chemotherapy as a consequence of surgery. Neoadjuvant therapy could have the additional advantages of identifying patients with rapid progression who would not benefit from surgical intervention; treating occult metastases earlier; and downsizing the tumors to increase the chance for a margin-free $(R 0)$ resection ${ }^{6}$. While the combination of surgery plus systemic therapy results in significant benefit relative to surgery alone, a subset of PDACs is highly resistant to systemic therapy. Nearly $40 \%$ of patients receiving surgery plus gemcitabine monotherapy experience relapse within one year of surgery $^{2}$. Even in the subset of fit patients who are candidates for more aggressive chemotherapy regimens, over $25 \%$ relapse within one year ${ }^{3}$. Currently, identifying this chemotherapy-resistant cohort prior to treatment remains a challenge, since conventional imaging, liquid biopsy, and molecular biomarkers are lacking.

The gene-expression subtypes defined in prior research ${ }^{7-9}$ potentially provide some guidance to this problem. The consensus subtypes have been termed classical, basal (also referred to as quasi-mesenchymal), and exocrine, terms chosen to reflect the normal cell types that most 


\section{Detecting Drug-Resistant PDAC}

closely correspond to the cancer cells. In retrospective evaluations of outcomes following curative resection, tumors with transcriptome profiles matching with the classical subtype had longer survival than the others ${ }^{7-9}$. Likewise, among patients with metastatic PDAC, the classical subtype was associated with longer survival in retrospective analyse ${ }^{10,11}$. On the other hand, patients with the classical subtype demonstrated no benefit from adjuvant chemotherapy ${ }^{9,12}$, in contrast to patients with the basal type, and cell lines of the classical subtype are more resistant to chemotherapy than those of the basal type ${ }^{8}$. Therefore, the predictive role of molecular subtyping in PDAC treatment remains to be established.

Further exploration of the role of molecular subtyping in PDAC requires a practical biomarker. Glycans (which are oligosaccharides) are an intriguing option, given their abundance on cell surfaces and secreted proteins, and because specific structures are indicators of cell differentiation. Glycans altered in pancreatic cancer include CA19-9 ${ }^{13}$, members of the Lewis blood group family ${ }^{14}$, and $\mathrm{ABO}$ blood group antigens ${ }^{14}$.

We recently identified a new biomarker of PDAC that is a cell-surface and secreted glycan called STRA (sialylated tumor-related antigen) ${ }^{15,16}$. The glycan is elevated in about half of the cases that do not show elevated CA19-9. A combination blood test using CA19-9 and the new marker performed better than CA19-9 alone in blinded tests for distinguishing PDAC from benign diseases ${ }^{17}$. The tumors expressing primarily sTRA tended to be sparse, poorly differentiated, or highly vacuolated, while those expressing mainly CA19-9 were part of well differentiated or moderately differentiated secretory glands ${ }^{16}$. These facts suggested that the two groups represent distinct subtypes of tumors having differing biology and clinical behavior. Of particular interest was the possibility that the drug-resistant group observed in clinical care corresponds to a glycan-defined subtype. We explored that possibility using multiple model systems and cohorts of clinical specimens. 
Detecting Drug-Resistant PDAC

\section{Materials and Methods}

\section{Cell Culture and Reagents}

The PaTu-8988S and PaTu8988T cell lines were obtained from Creative Bioarray (Shirley, NY), and Colo357, L3.3, and L3.6PL lines were kindly provided by Dr. Isaiah J. Fidler (University of Texas, MD Anderson Cancer Center). The remaining cell lines were obtained from ATCC (Manassas, VA). All cell lines were cultured in RPMI-1640 supplemented with $5 \%$ fetal bovine serum, $2 \mathrm{mM} \mathrm{L-Glutamine,} \mathrm{and} 100 \mathrm{IU} / \mathrm{mL}$ penicillin/streptomycin. The cells were grown at 37 ${ }^{\circ} \mathrm{C}$ in a humidified atmosphere supplemented with $5 \%(\mathrm{v} / \mathrm{v}) \mathrm{CO}_{2}$. The chemotherapeutic reagents cisplatin, etoposide, gemcitabine, and 5-FU were obtained from Sigma (St. Louis, MO). Irinotecan, oxaliplatin, and paclitaxel were obtained from Cayman Chemical (Ann Arbor, MI). All drugs were dissolved in dimethyl sulfoxide or dimethylformamide. For the preparation of

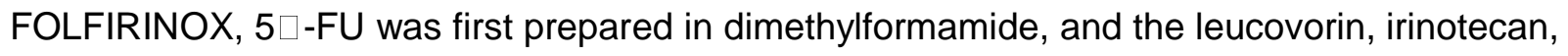
and oxaliplatin were each added in a 1:5 ratio by weight to $5 \square-\mathrm{FU}$. The concentration of FOLFIRINOX was calculated based on the $5 \square-F U$ concentration.

\section{Drug Treatment Studies}

Cells were seeded into 96 well plates at $2 \times 10^{3}$ cells per well and cultured for $3 \mathrm{~d}$ before treatment with a drug or drug mixture at six different concentrations each. After $3 \mathrm{~d}$, cell viability was estimated using CellTiter-Glo (Promega, Madison, $\mathrm{WI}$ ). The $\mathrm{IC}_{50}$ values were calculated using GraphPad Prism 6 (GraphPad Software, San Diego, CA) with 5-parameter, variable-slope fits.

For the outgrowth of drug-resistant L3.3 sublines, the cells were cultured in medium containing cisplatin $(2 \mu \mathrm{M})$, etoposide $(2 \mu \mathrm{M})$, gemcitabine $(0.02 \mu \mathrm{M})$, or $5-\mathrm{FU}(5 \mu \mathrm{M})$ for $3 \mathrm{~d}$ and recovered for $4 \mathrm{~d}$ in medium without drug. This process was repeated for three rounds, after which the cells were maintained in a $1 / 10$ of concentration of the drug used for selection. 


\section{Detecting Drug-Resistant PDAC}

\section{Statistics}

Differences between marker-positive and marker-negative cell lines in $\mathrm{IC}_{50}$ and percent viability were tested using the Mann-Whitney $U$ test. Differential expression in the RNAseq data was tested using empirical Bayes quasi-likelihood F-tests, and the p values were adjusted using the Benjamini-Hochberg method. Differences in OS between patient groups in the survival analyses were evaluated with the log-rank test. Differences between patient groups in proportions of patients with long or short OS were analyzed with the Fisher's Exact test and the Breslow-Day test for homogeneity of odds ratios. Differences in sensitivity and the average of sensitivity and specificity were analyzed with the Wald test based on bootstrap SE estimate. P values of less than 0.05 were considered significant.

To perform cross validation with bootstrapping, the patients were randomly divided into five groups stratified on case-control status. Four groups served as the training subset to derive the panel and one group served as the testing subset to estimate the panel performance. The five estimates of performance were then averaged to get the final performance estimate. This crossvalidation was repeated on 200 samplings of the cohort to compute the standard error for the cross-validated performance estimate.

\section{Human Specimens}

The plasma samples were collected under protocols approved by the Institutional Review Boards at the University of Pittsburgh Medical Center and the Medical College of Wisconsin. The tissue samples for tissue microarrays were collected under approved protocols at the Medical University of South Carolina, University of Pittsburgh Medical Center, and Memorial Sloan Kettering Cancer Center. All subjects provided written, informed consent, and all methods were performed in accordance with an assurance filed with and approved by the U.S. Department of Health and Human Services. 


\section{Detecting Drug-Resistant PDAC}

The plasma collections took place prior to any surgical, diagnostic, or medical procedures. The donors consisted of patients with pancreatic cancer or a benign condition involving the pancreas, and from healthy subjects. All blood samples (EDTA plasma) were collected according to the standard operating procedure from the Early Detection Research Network and were frozen at $-70^{\circ} \mathrm{C}$ or colder within $4 \mathrm{~h}$ of time of collection. Aliquots were shipped on dry ice and thawed no more than three times prior to analysis. Disease progression was diagnosed radiographically based on CT scans performed at 3-4 month intervals for the first two years and at six month intervals thereafter. Occasionally, a tissue biopsy was performed. 


\section{Detecting Drug-Resistant PDAC}

\section{Results}

We initially tested for differences between PDACs classified by glycan expression using a panel of 27 cell lines. We classified each cell line based on the sTRA glycan or the CA19-9 glycan

(Figure 1A). Both glycans are capped with sialic acid on type-1 N-acetyl-lactosamine (LacNAc), the disaccharide of galactose linked $\beta 1,3$ to $N$-acetyl-glucosamine(GlcNAc), and the CA19-9 epitope has a fucose attached to the $\mathrm{N}$-acetyl-glucosamine, which is necessary for its recognition by selectin receptors. Type- 1 LacNAc, as recognized by the TRA-1-60 antibody ${ }^{18}$, is a marker for induced pluripotent stem cells, but the sialylated version has not been well studied due to lack of an effective antibody. We indirectly detected the sialylated structure using sialidase to uncover the TRA-1-60 epitope (Figure 1A).

The cell-surface expression of STRA and CA19-9 was variable among the cell lines, with some primarily expressing only one glycan and others expressing both or neither (Figs. 1B and 1C). Organoid models of pancreatic cancer ${ }^{19,20}$ likewise showed variable expression of one, both, or neither of the glycans (Figure 1D), with slightly different proportions among them (Figure 1E). The difference in proportions could be due to cellular selection, effects of culture conditions, or merely random variation within the small sample size. Nevertheless, these two model systems agree in showing distinct expression of the glycans.

\section{Gene-expression programs distinguishing the glycan-defined subtypes}

Using the cell lines and organoids, we then asked whether similar differences exist in gene transcription programs. A total of 267 genes were differentially expressed between the sTRAexpressing cell lines (not including the three lines also expressing CA19-9) and all others (Figure 2A and Supplemental Table 1). No individual genes were differentially expressed between the CA19-9 and STRA groups at $p<0.05$ after multiple-hypothesis correction, possibly due to the lower number of CA19-9-positive lines. The sTRA-associated genes had ontologies that were enriched in developmental, drug metabolism, and glycan-biosynthesis pathways 


\section{Detecting Drug-Resistant PDAC}

(Figure 2A). The developmental gene BMP4 was a strong individual marker of sTRA cells, as was CYP3A5 (Figure 2A), a gene previously identified as a marker of PDACs identified as classical and exocrine ${ }^{21}$. In addition, 9 of 14 sTRA-expressing lines were identified as classical,

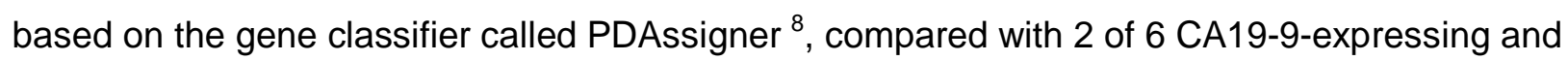
0 of 10 glycan-negative lines (Figure 2A), suggesting that sTRA is more likely to recognize the classical subtype. Gene-set enrichment analysis showed that sets defining stem-like differentiation, stem-like metabolism, and the classical subtype were enriched in the sTRAexpressing cells (Figure 2B and Supplemental Table 1). Among individual genes that have been proposed as markers of class, the expressions of GATA6 and CYP3A5 were higher in the sTRA cells (Figure 2C). KRT81 and HNF1A showed weak associations with sTRA.

The epithelial/mesenchymal state of cancer cells has been widely explored as an indicator of their origin, invasiveness, or overall tumor-forming aggressiveness. All six of the CA19-9expressing cell lines were epithelial, as determined by the gene expression of Zeb-1 and Ecadherin (Figure 2C) and by morphology (Supplemental Figure 1), but the sTRA-expressing cells and those expressing neither glycan were of various types (Figure $2 \mathrm{C}$ ). The organoid models all had epithelial morphologies, but three of the models had mesenchymal characteristics (high Zeb-1 and low E-cadherin) by gene expression (not shown). Two of these produced sTRA exclusively and the third produced neither glycan. The data from both model systems suggest that some sTRA-expressing cancer cells have the potential for mesenchymallike differentiation, in contrast to CA19-9-expressing cells.

The organoid models were dissimilar to the cell lines in their overall gene expression profiles. All but one of the organoids had classical subtype expression by PDAssigner. However, the gene sets used to divide the organoids ${ }^{20}$ into $\mathrm{C} 1 / \mathrm{C} 2$ and classical/basal groups did not separate the cell lines into similar groups (not shown). This finding could be because all of the organoids were epithelial, or it could derive from differences in culture conditions. 


\section{Detecting Drug-Resistant PDAC}

The type of KRAS mutation in cancer potentially can drive differences in phenotype ${ }^{22}$. The lesscommon Q61 alteration appeared exclusively in the cell lines and organoids that expressed only sTRA (Figure 2D). The G12V mutation was in 3 of 11 cell lines and 1 of 3 organoids that expressed only sTRA, in comparison to 0 of 3 cell lines and 1 of 8 organoids that expressed only CA19-9. The common G12D mutation and the rare wild-type cases were found throughout the subtypes. While these observations are based on relatively small sample sizes, they suggest that the $\mathrm{Q} 61 \mathrm{H} / \mathrm{R}$ mutation fosters cancers that express sTRA in the absence of CA19-9. Mutations in other cancer-associated genes were not more frequent in either glycan-defined group (not shown).

\section{Resistance to chemotherapy in STRA-high cultures}

We determined the resistance of the 27 cell lines to eight chemotherapeutics that are either front-line or alternative treatments against pancreatic cancer. In a single-dose study, the sTRAexpressing cell lines were more resistant than the sTRA-negative cell lines in each case (Figure 3A). In dose-response analyses to obtain the $\mathrm{IC}_{50}$ concentrations (Figure $3 \mathrm{~B}, 3 \mathrm{C}$, and Supplemental Figure 2), the sTRA cells were significantly more resistant than the non-sTRA cells ( $p<0.05$, Mann-Whitney U Test) to 6 of the 8 drugs. For gemcitabine, the resistance was higher in the sTRA group but with less statistical significance $(p=0.07$, Mann-Whitney U Test); for oxaliplatin, resistance was similar between the groups. In contrast, CA19-9 did not define a resistant group (Supplemental Figure 2). Two outlier lines that expressed sTRA but were sensitive-PSN-1 and CFPAC-1-had mutations that were not consistent with the other sTRApositive cells lines (Figure 2D).

We asked whether the high resistance corresponded to traits that have been associated with resistance. The STRA-positive lines had higher levels of drug-metabolizing enzymes from the cytochrome P450 family ( $p<0.05$, Mann-Whitney $U$ test), and they had trends toward higher 


\section{Detecting Drug-Resistant PDAC}

levels of the stem marker ALDH1A3 and longer doubling times (Figure 3D). Thus, some sTRA lines have resistance traits, but the mechanism of resistance may differ between cell lines.

We further tested the above relationships using sets of isogenic cell lines, where all cell lines in a set are from the same individual (Supplement Figure 3). To generate sublines with increased drug resistance, we repeatedly cultured the L3.3 cell line in sublethal concentrations of each of four drugs, followed by recovery and outgrowth of the surviving cells. We found that sTRA expression was increased in several of the sublines, both in the percentage of stained cells and in total staining intensity. Genotyping across over 15,000 SNPs confirmed agreement in genotype between the sublines (not shown). Consistent with the results across diverse cell lines, the sublines with higher sTRA coincided with significantly increased resistance to the drugs.

The cell lines PaTu8988S and PaTu8988T were derived from a single patient and also showed differences in sTRA. The PaTu8988S cell line had much higher sTRA (Supplemental Figure 3) (neither expressed CA19-9), and in accord with the results above, it showed higher resistance to 3 of 4 chemotherapies. These findings from isogenic cell lines parallel the findings from the panel of cell lines and support the idea that sTRA is a biomarker for a subtype of PDAC having high drug resistance.

\section{Predictive value of the STRA levels in primary tumors}

Next, we tested whether the sTRA levels in primary tumors are associated with resistance to systemic chemotherapy. We determined STRA levels in two ways, by a gene-expression classifier and by immunofluorescence. To develop a gene-expression classifier, we identified the significantly up-regulated or down-regulated genes $(p<0.02$, Bayes quasi-likelihood F-test after multiple-testing correction) associated with sTRA expression in the panel of 27 cell lines (Supplemental Table 1) and used the algorithm from PDAssigner ${ }^{8}$ to assign classes. We used this algorithm because of its previous robust performance and its simplicity for adoption with 


\section{Detecting Drug-Resistant PDAC}

new gene sets. We applied the classifier to 150 cases of PDAC from The Cancer Genome Atlas $^{23}$ and to 180 cases from the International Cancer Genome Consortium ${ }^{24}$ that had survival information. In both cohorts, distinct groups of patients showed overall differences in expression between genes associated with high sTRA and those associated with low sTRA (Figure 4A). Tests of group differences in central tendency using the classifier genes showed significance ( $p$ $=0.001$, Adonis test, Vegan R package). This finding confirmed the consistency between the cell lines and both cohorts in the differential expression of the gene groups, and it supports the idea that the classifier identifies true subtypes rather than random variation in expression patterns.

We assigned the patients to an sTRA or non-sTRA group based on the median of the calculated score of the classifier. No difference in overall survival (OS) was evident between the sTRA and non-sTRA groups of patients, but among the patients assigned to the non-sTRA group, those receiving adjuvant therapy had significantly longer overall survival (OS) than those who did not ( $p<0.001$, Log-rank test) (Figure 4B). Among the patients assigned to the sTRA group, no difference was observed. Both the TCGA and ICGC data sets showed this relationship. Other classifiers for the classical subtype gave similar results (Figure $4 \mathrm{C}$ ), but not as consistently between data sets as the sTRA classifier.

In a parallel approach, we asked whether the directly measured amount of sTRA in primary tumors associated with a lack of response to adjuvant therapy. We used multimarker immunofluorescence ${ }^{16,25}$ to quantify sTRA and CA19-9 in tissue microarrays (TMAs) that included tumors from patients who had long ( $>3$ years) or short $(<1$ year) OS following surgery, and who either did or did not receive adjuvant therapy (Supplemental Table 2 and ref. ${ }^{26}$ ). We quantified the markers using previously developed software that enables unbiased, automated quantification of multimarker IF data ${ }^{16,27}$ (Figure 5A and Supplemental Table 2). The sTRA and CA19-9 levels showed little correlation with each other (Figure 5B), consistent with the two 


\section{Detecting Drug-Resistant PDAC}

markers indicating different groups of tumors. The sTRA levels, but not the CA19-9 levels, were higher in the short-OS group ( $p=0.0077$, Wilcoxon Rank-Sum test) (Figure 5C). Likewise, in receiver-operator characteristic analysis to distinguish long from short OS, a test for area-underthe-curve $(A \cup C)$ unequal to 0.5 was statistically significant using sTRA ( $p=0.008$, Wilcoxon test), but not using CA19-9 (Figure 5D).

The TMAs included similar proportions of long and short OS in the adjuvant and no-adjuvant groups (Figure 5E). Among the patients receiving adjuvant chemotherapy, those with high sTRA showed a significantly lower proportion of long OS than those with low sTRA ( $p=0.003$, Fisher's Exact test). Among the patients with high sTRA, those receiving neoadjuvant chemotherapy had a significantly lower proportion of long OS than those not receiving neoadjuvant chemotherapy ( $p=0.01$, Fisher's Exact test). No other comparison showed a significant difference. In addition, the Breslow-Day test for homogeneity of odds ratios (for association between survival and therapy) across biomarker-defined subgroups was highly significant for sTRA $(p=0.006)$ but was not significant for CA19-9 $(p=0.18)$. These results indicate a differential effect of adjuvant therapy on patient survival between the sTRA-high and sTRA-low groups.

Another set of TMAs contained samples from tumors that had been exposed to neoadjuvant therapy ${ }^{16}$. Although these TMAs did not reveal significant associations between survival and the individual marker amounts, possibly owing to changes in total glycan levels induced by the chemotherapy, they did show relationships between survival and relative biomarker abundance. The tumors that were dominant in cells producing only sTRA or only CA19-9, or that contained both types of single-labeled cells, were in the short survival group, with few exceptions (Supplemental Figure 4 and Supplemental Table 2). This relationship showed a connection between poor outcome and the outgrowth following neoadjuvant therapy of STRA-dominant or CA19-9-dominant clones. 


\section{Detecting Drug-Resistant PDAC}

\section{Predicting rapid relapse using a blood test}

A biomarker that is measurable in blood samples would have higher clinical utility than one accessible only from tissue, given the difficulties in sampling tissue from the pancreas. We therefore examined whether secreted glycans could be used as indicators of cellular expression. We measured the soluble levels of sTRA glycan using sandwich immunoassays, in which we detect sTRA on the proteins captured by one of three different capture antibodies (Figure 6A). The agreement between the cell-surface expression and the amount of glycan in the conditioned medium was high, with 25 of the 27 cell lines matching between cell-surface glycan expression and detection in the conditioned medium (Supplemental Figure 5). The agreement was also good for the organoids: 23 of 27 matched between cell-surface and conditioned-medium amounts for both sTRA and CA19-9. Furthermore, in a previous study we found that the peripheral blood glycans correlated with tumor glycans for cell-line xenograft mouse models, patient-derived xenograft mouse models, and human PDAC patients ${ }^{16}$. Thus, the secreted levels and blood levels are good indicators of the tumor expression of the glycans.

This relationship, combined with the above findings from cell culture and primary tumors, presented the possibility that high plasma sTRA identifies PDACs that do not benefit from systemic chemotherapy. We investigated this possibility among subjects who were beginning neoadjuvant therapy. Such a cohort would best reveal differences in resistance to chemotherapy, while avoiding potentially confounding major variations in the extent of disease or treatment history.

We measured STRA and CA19-9 in plasma samples that had been acquired prior to the start of neoadjuvant therapy, first in a discovery set and then in a test set (data in Supplemental Table 3). We asked whether any of the biomarkers could serve as an indicator of short time-toprogression (TTP), as defined by radiographic evidence of progression. We dichotomized the 


\section{Detecting Drug-Resistant PDAC}

patients using a cutoff of 18 months from the time of diagnosis, based on the approximate rate of $50 \%$ recurrence within one year after the completion of treatments.

In the discovery set, two of the sTRA immunoassays were significantly higher in subjects with short TTP than in subjects with long TTP (Figure 6B). CA19-9 did not show an association with TTP, nor did age or sex of the patients (Supplemental Table 3). We used a multi-marker classifier system called $\mathrm{MSS}^{28}$ to develop a three-marker panel. Patients elevated in two or more of the sTRA assays (using thresholds optimized for each marker, Figure 6B) were especially likely to have short TTP, as 16 of 17 had short TTP (94\% PPV) (Figure 6C and Table 1). This result translated to $98 \%$ specificity (41/42 with long TTP were high in 1 or less) and $46 \%$ sensitivity (16/35 with short TTP were high in 2 or more). For CA19-9, a threshold that gave $95 \%$ specificity gave only about $3 \%$ sensitivity. To minimize the effect of overfitting on the estimate of panel performance, we further assessed the panel performance using five-fold cross-validation with 200-fold bootstrapping (re-samplings of the cohort). The improvements in cross-validated sensitivity and the average of sensitivity and specificity were statistically significant $(p<0.0001$, Wald test based on bootstrap SE estimate) (Table 1).

This is a provisional result, because the panel was optimized based on the data from the discovery set. We then applied the panel to a blinded test set. We applied the thresholds that were derived from the discovery set to the test set and made case/control calls on the blinded samples. The calls were sent to the collaborators who collected the samples, and upon comparison with the true outcomes data, the result was $96 \%$ specificity (16/35 with short TTP were high in 2 or more) and 56\% sensitivity (15/27 with long TTP were high in 1 or less) (Table 1). The improvements in sensitivity and the average of sensitivity and specificity were statistically significant $(p<0.0001$, Wald test based on 1000-fold bootstrap SE estimate, Table 1). We tested adjustments to the individual marker thresholds, considering that the thresholds were trained on a relatively small cohort, and we found that the optimized thresholds (naïve 


\section{Detecting Drug-Resistant PDAC}

performance) gave 94\% PPV (17/18 with two or more marker elevations had short TTP), 96\% specificity (27/28 with long TTP), and 63\% sensitivity (17/27 with short TTP) (Figure 6D and Table 1). Bootstrap analysis with cross validation confirmed the statistical significance of the improvement (Table 1). The three individual sTRA assays showed strong associations with short TTP ( $p=0.008$ to 0.00008 , Mann-Whitney U test), as did CA19-9 ( $p=0.007)$ (Supplemental Figure 6).

The sTRA panel also identified differences in TTP in Kaplan-Meier analysis (Figure 6E). KaplanMeier analysis is appropriate here because the cohort is a random selection of the patients seen in the clinic. In both sets, patients positive in the panel (elevated in 2 or more of the sTRA assays) had shorter TTP than the rest of the patients. In the test set, the difference was highly significant $(p<0.0001$, log-rank test) for sTRA and moderately associated $(p=0.04)$ for CA19-9 (significance not evaluated in the discovery set).

To further test these associations, we obtained outcomes for a subset of patients in a previous study of these markers ${ }^{17}$ who had received neoadjuvant therapy (data in Supplemental Table 3). The study was not designed for this question but nevertheless could provide insights. In two separate cohorts, two of the sTRA assays trended with short overall survival $(p=0.05$ and 0.06 , Supplemental Figure 6). CA19-9 showed no such trend in either cohort. These findings substantiate the use of a blood test for sTRA to identify a subtype of pancreatic cancers that is resistant to chemotherapy. 


\section{Detecting Drug-Resistant PDAC}

\section{Discussion}

This research demonstrates the use of the sTRA glycan to identify the PDAC cases that are highly resistant to chemotherapy. We used both cellular and blood-based immunofluorescence assays, as well as a surrogate gene-expression signature, to identify resistant PDAC. The identification was consistent across sample types—cell-culture models, isogenic cell lines, primary tumors, and blood plasma samples_-and it was consistent in both the adjuvant and neoadjuvant settings. The immediate implication of this result relates to the development of treatment plans for patients with resistant PDAC. For patients with resectable PDAC, potentially morbid operations could be avoided if rapid relapse following surgery could be predicted a priori. For patients with metastatic disease and patients undergoing neoadjuvant therapy, a practical biomarker could guide the choices and comparisons of the treatment options. For example, FOLFIRINOX is suggested to be slightly better than gemcitabine for the classical subtype ${ }^{10,29}$, possibly indicating a difference between the sTRA-positive and STRA-negative types. Patient stratification also could improve the selection of patients that receive nab-paclitaxel ${ }^{5}$. Furthermore, the sTRA assay could be used in subgroup analyses of the many human trials currently underway, given that many trials do not meet primary objectives but show evidence of efficacy in subgroups. Trials could involve targeted therapies suggested from studies on the cell-culture and organoid models that are available for sTRA-positive and sTRA-negative PDACs. Thus, our biomarker has value for patient stratification using current options, as well as for research using model systems and in biomarker-guided drug trials.

A blood test has particular value in the clinical setting because physicians could stratify patients prior to any treatments, without a biopsy. A blood test also could extend the analyses of biomarker-based stratification beyond only the patients who have resected tumors or biopsy samples available. Furthermore, a blood test would capture secretions from the whole tumor rather than just the cells that are sampled by a biopsy, which may not reflect the heterogeneity 


\section{Detecting Drug-Resistant PDAC}

of the tumor. Various blood tests have potential value for detecting or diagnosing PDAC, including mutated DNA in the circulation ${ }^{30,31}$, tumor exosomes, and metabolites ${ }^{32-34}$, but they do not predict therapeutic responses. Highly elevated CA19-9 in the blood and the failure to drop to normal levels following neoadjuvant therapy or surgery ${ }^{35,36}$ are unfavorable prognostic factors ${ }^{37}$, but CA19-9 does not indicate a distinct subtype or predict resistance to chemotherapy ${ }^{35}$. The sTRA blood test, in these initial studies, performed at a level that warrants further investigation as a biomarker to select for neoadjuvant or adjuvant chemotherapy. Blinded, prospective studies using a clinical assay will be required to fully assess the value of this test to patients and physicians.

If tissue is available from biopsy or resection, several cellular markers in addition to sTRA have the potential to be predictive. For example, high levels of the human equilibrative nucleoside transporter 1 (hENT1) showed a relationship with longer survival among patients receiving adjuvant gemcitabine ${ }^{38}$, presumably based on its role in the transport of gemcitabine and other drugs into cells. Immunohistochemical detection of KRT81 and HNF1A correlated with the basal and exocrine subtypes, respectively, and CYP3A5 indicated the exocrine subtype ${ }^{21}$. The analysis of immunohistochemistry data from TMAs indicated that high KRT81 corresponds to worse prognosis than high $\mathrm{HNF} 81^{29}$, in line with the associations made by gene-expression profiling. GATA6 is an indicator of the classical subtype ${ }^{7,8}$ and has been detected by in-situ hybridization in biopsy specimens ${ }^{10}$. Using IHC detection of GATA6 in TMAs, researchers found that tumors with high GATA6 had better prognoses than those with low GATA6, and that GATA6 had a weak association with resistance to $5 \mathrm{FU}^{39}$. These markers show promise, but their value for patient stratification will need to be validated in prospective studies.

This work extends previous findings relating to the prognostic and predictive value of the molecular subtypes. The classical subtype in previous research indicated a better prognosis than the basal subtype, but, on the other hand, it tended to benefit less from chemotherapy ${ }^{9,12}$. 


\section{Detecting Drug-Resistant PDAC}

This result is also consistent with an in vitro study in cultured cells, which indicated that classical-subtype cells were more resistant to gemcitabine ${ }^{8}$. But the relative value for prognosis versus treatment prediction was not clear, and a recent study suggested that the classical subtype is more sensitive to chemotherapy than the basal subtype ${ }^{11}$. The differences between the studies may result from several sources. The latter study included non-resectable, advanced PDAC (the COMPASS Trial), while the former studies involved resectable PDAC. Advanced cancer could be less responsive in general than localized disease. The COMPASS study also used a different classifier that was more stringent than the original, and the study was not designed to distinguish prognostic from predictive value, since it did not include a non-treatment control group. Overall, the results indicate that native prognosis and sensitivity to chemotherapy are not necessarily linked, and that the classical subtype is suboptimal for decoupling these traits.

The sTRA subtype seems to distinguish the traits better: it was indicative of chemotherapy resistance, but not of a poor prognosis. It encompassed resistant cancers of both subtypes and was more consistent than the classical/basal system in identifying resistance in the primary tumors. A valid model is that the sTRA subtype more precisely identifies resistant tumors, but the classical subtype could be more effective for stratifying by native prognosis. Ultimately, the typing of cancers and prediction of drug responses could involve both glycans and other types of markers. Further studies should focus on clarifying additional markers that are suggestive of other subtypes. The genes HNF1A, CDH17, LGALS4, and CYP3A5 have been variously assigned as markers of non-basal subtypes including exocrine and classical but without good agreement between studies ${ }^{40}$. The elevation of these genes in most sTRA cancers could indicate that a third subtype is at least partially encompassed by sTRA.

The sources of treatment resistance in the sTRA-positive cancers potentially could be discerned from the model systems and gene-expression studies, along with directions to test for new 


\section{Detecting Drug-Resistant PDAC}

options. The sTRA cells could derive from a stem-cell population, given the structural similarity between the sTRA glycan and the glycan marker of iPSCs, as well as the stem-like gene expression. The development of resistant clones following therapy also is thought to arise from clonal diversity within tumors ${ }^{41}$, and stem-like cancer cells could be a minority subpopulation in early stages but become dominant in resistant cancers. Such a trajectory could underlie the development of tumors that are dominant in STRA (Figure 5). Future studies could track changes in glycan markers in relation to clonal diversity.

Building on these findings, our next steps will involve the validation of clinical assays for prospective studies and the analyses of model systems in order to understand the susceptibilities of sTRA-positive cancers. Based on the gene-expression results, a successful path may involve metabolic approaches ${ }^{42}$. Alternatively, probing the sTRA-positive subtype for dependencies on particular nutrient sources may be feasible. These directions in research are made possible because we now have a practical assay to detect chemoresistant PDAC using either tissue or plasma. The use of such an assay in model systems, and then in clinical specimens to detect and follow the resistant subtype, should help both the development and the application of new treatments against PDAC. 


\section{Detecting Drug-Resistant PDAC}

\section{Acknowledgements}

We thank the core services at the Van Andel Institute for expert support of this research, particularly the Optical Imaging, Bioinformatics and Biostatistics, Genomics, and Pathology and Biorepository cores. We thank Zachary Klamer, MS, at the Van Andel Institute for support in the processing and analysis of the plasma biomarker data; Toshinori Hinoue, PhD, at the Van Andel Institute for advising on the analysis of TCGA and ICGC datasets, and Christine Decapite at the University of Pittsburgh Medical Center for assistance with data coordination. 


\section{Detecting Drug-Resistant PDAC}

\section{References}

1. Neoptolemos JP, Kleeff J, Michl P, et al. Therapeutic developments in pancreatic cancer: current and future perspectives. Nat Rev Gastroenterol Hepatol 2018;15:333-348.

2. Oettle $H$, Post $S$, Neuhaus $P$, et al. Adjuvant chemotherapy with gemcitabine vs observation in patients undergoing curative-intent resection of pancreatic cancer: $a$ randomized controlled trial. JAMA 2007;297:267-77.

3. Conroy T, Hammel P, Hebbar M, et al. FOLFIRINOX or Gemcitabine as Adjuvant Therapy for Pancreatic Cancer. N Engl J Med 2018;379:2395-2406.

4. Neoptolemos JP, Palmer DH, Ghaneh P, et al. Comparison of adjuvant gemcitabine and capecitabine with gemcitabine monotherapy in patients with resected pancreatic cancer (ESPAC-4): a multicentre, open-label, randomised, phase 3 trial. Lancet 2017;389:10111024.

5. Von Hoff DD, Ervin T, Arena FP, et al. Increased survival in pancreatic cancer with nabpaclitaxel plus gemcitabine. N Engl J Med 2013;369:1691-703.

6. Asare EA, Evans DB, Erickson BA, et al. Neoadjuvant treatment sequencing adds value to the care of patients with operable pancreatic cancer. J Surg Oncol 2016;114:291-5.

7. Bailey P, Chang DK, Nones K, et al. Genomic analyses identify molecular subtypes of pancreatic cancer. Nature 2016;531:47-52.

8. Collisson EA, Sadanandam A, Olson P, et al. Subtypes of pancreatic ductal adenocarcinoma and their differing responses to therapy. Nat. Med. 2011;4:500-503.

9. Moffitt RA, Marayati R, Flate EL, et al. Virtual microdissection identifies distinct tumorand stroma-specific subtypes of pancreatic ductal adenocarcinoma. Nat Genet 2015;47:1168-78.

10. Aung KL, Fischer SE, Denroche RE, et al. Genomics-Driven Precision Medicine for Advanced Pancreatic Cancer: Early Results from the COMPASS Trial. Clin Cancer Res 2018;24:1344-1354.

11. O'Kane GM, Grunwald BT, Jang GH, et al. GATA6 expression distinguishes classical and basal-like subtypes in advanced pancreatic cancer. Clin Cancer Res 2020;epub ahead of print.

12. Collisson EA, Bailey $\mathrm{P}$, Chang DK, et al. Molecular subtypes of pancreatic cancer. Nat Rev Gastroenterol Hepatol 2019;16:207-220.

13. Tempero MA, Uchida E, Takasaki H, et al. Relationship of carbohydrate antigen 19-9 and Lewis antigens in pancreatic cancer. Cancer Res 1987;47:5501-3.

14. Pour PM, Tempero MM, Takasaki $\mathrm{H}$, et al. Expression of blood group-related antigens $A B H$, Lewis $A$, Lewis $B$, Lewis $X$, Lewis $Y$, and CA 19-9 in pancreatic cancer cells in comparison with the patient's blood group type. Cancer Res 1988;48:5422-6.

15. Tang H, Partyka K, Hsueh P, et al. Glycans related to the CA19-9 antigen are elevated in distinct subsets of pancreatic cancers and improve diagnostic accuracy over CA19-9. Cell Mol Gastroenterol Hepatol 2016;2:201-221 e15.

16. Barnett D, Liu Y, Partyka K, et al. The CA19-9 and Sialyl-TRA Antigens Define Separate Subpopulations of Pancreatic Cancer Cells. Sci Rep 2017;7:4020. 


\section{Detecting Drug-Resistant PDAC}

17. Staal B, Liu Y, Barnett D, et al. The sTRA Plasma Biomarker: Blinded Validation of Improved Accuracy over CA19-9 in Pancreatic Cancer Diagnosis. Clin Cancer Res 2019;29:2745-2754.

18. Andrews PW, Banting G, Damjanov I, et al. Three monoclonal antibodies defining distinct differentiation antigens associated with different high molecular weight polypeptides on the surface of human embryonal carcinoma cells. Hybridoma 1984;3:347-61.

19. Boj SF, Hwang C, Baker LA, et al. Organoid Models of Human and Mouse Ductal Pancreatic Cancer. Cell 2014;160:324-338.

20. Tiriac H, Belleau P, Engle DD, et al. Organoid Profiling Identifies Common Responders to Chemotherapy in Pancreatic Cancer. Cancer Discov 2018;8:1112-1129.

21. Noll EM, Eisen C, Stenzinger A, et al. CYP3A5 mediates basal and acquired therapy resistance in different subtypes of pancreatic ductal adenocarcinoma. Nat Med 2016;22:278-87.

22. Hamidi $\mathrm{H}$, Lu M, Chau K, et al. KRAS mutational subtype and copy number predict in vitro response of human pancreatic cancer cell lines to MEK inhibition. Br J Cancer 2014;111:1788-801.

23. Biankin AV. Integrated Genomic Characterization of Pancreatic Ductal Adenocarcinoma. Cancer Cell 2017;32:185-203.e13.

24. Connor AA, Denroche RE, Jang GH, et al. Association of Distinct Mutational Signatures With Correlates of Increased Immune Activity in Pancreatic Ductal Adenocarcinoma. JAMA Oncol 2017;3:774-783.

25. Gerdes MJ, Sevinsky CJ, Sood A, et al. Highly multiplexed single-cell analysis of formalinfixed, paraffin-embedded cancer tissue. Proc Natl Acad Sci U S A 2013;110:11982-7.

26. Winter JM, Tang LH, Klimstra DS, et al. A novel survival-based tissue microarray of pancreatic cancer validates MUC1 and mesothelin as biomarkers. PLOS ONE 2012;7:e40157.

27. Barnett D, Hall J, Haab B. Automated Identification and Quantification of Signals in Multichannel Immunofluorescence Images: The SignalFinder Platform. Am J Pathol 2019;189:1402-1412.

28. Fallon BP, Curnutte B, Maupin KA, et al. The Marker State Space (MSS) Method for Classifying Clinical Samples. PLoS ONE 2013;8:e65905.

29. Muckenhuber A, Berger AK, Schlitter AM, et al. Pancreatic Ductal Adenocarcinoma Subtyping Using the Biomarkers Hepatocyte Nuclear Factor-1A and Cytokeratin-81 Correlates with Outcome and Treatment Response. Clin Cancer Res 2018;24:351-359.

30. Cohen JD, Javed AA, Thoburn C, et al. Combined circulating tumor DNA and protein biomarker-based liquid biopsy for the earlier detection of pancreatic cancers. Proc Natl Acad Sci U S A 2017;114:10202-10207.

31. Cohen JD, Li L, Wang Y, et al. Detection and localization of surgically resectable cancers with a multi-analyte blood test. Science 2018;359:926-930.

32. Lennon AM, Wolfgang CL, Canto MI, et al. The Early Detection of Pancreatic Cancer: What Will It Take to Diagnose and Treat Curable Pancreatic Neoplasia? Cancer Res 2014;74:3381-9. 
33. Kelly KA, Hollingsworth MA, Brand RE, et al. Advances in Biomedical Imaging, Bioengineering, and Related Technologies for the Development of Biomarkers of Pancreatic Disease: Summary of a National Institute of Diabetes and Digestive and Kidney Diseases and National Institute of Biomedical Imaging and Bioengineering Workshop. Pancreas 2015;44:1185-94.

34. Young MR, Wagner PD, Ghosh S, et al. Validation of Biomarkers for Early Detection of Pancreatic Cancer: Summary of The Alliance of Pancreatic Cancer Consortia for Biomarkers for Early Detection Workshop. Pancreas 2018;47:135-141.

35. Tsai S, George B, Wittmann D, et al. Importance of Normalization of CA19-9 Levels Following Neoadjuvant Therapy in Patients With Localized Pancreatic Cancer. Ann Surg 2018;271:740-747.

36. Boone BA, Steve J, Zenati MS, et al. Serum CA 19-9 response to neoadjuvant therapy is associated with outcome in pancreatic adenocarcinoma. Ann Surg Oncol 2014;21:43518.

37. Nakagawa K, Akahori T, Nishiwada S, et al. Prognostic factors for actual long-term survival in the era of multidisciplinary treatment for pancreatic ductal adenocarcinoma. Langenbecks Arch Surg 2018;403:693-700.

38. Farrell JJ, Elsaleh H, Garcia M, et al. Human equilibrative nucleoside transporter 1 levels predict response to gemcitabine in patients with pancreatic cancer. Gastroenterology 2009;136:187-95.

39. Martinelli P, Carrillo-de Santa Pau E, Cox T, et al. GATA6 regulates EMT and tumour dissemination, and is a marker of response to adjuvant chemotherapy in pancreatic cancer. Gut 2017;66:1665-1676.

40. Martens S, Lefesvre $P$, Nicolle R, et al. Different shades of pancreatic ductal adenocarcinoma, different paths towards precision therapeutic applications. Ann Oncol 2019;30:1428-1436.

41. Seth S, Li CY, Ho IL, et al. Pre-existing Functional Heterogeneity of Tumorigenic Compartment as the Origin of Chemoresistance in Pancreatic Tumors. Cell Rep 2019;26:1518-1532.e9.

42. Halbrook CJ, Lyssiotis CA. Employing Metabolism to Improve the Diagnosis and Treatment of Pancreatic Cancer. Cancer Cell 2017;31:5-19. 


\section{Detecting Drug-Resistant PDAC}

\section{Figures}

Figure 1. Complementary glycan expression in model systems. A) Glycan structures. B) Diverse sTRA and CA19-9 expression in cell culture. C) Cell-surface expression of 27 cell lines. The classification of positivity at the bottom was based on a cutoff of signal-to-noise ratio > 3. D) Diverse glycan expression in organoid models. E) Cell-surface glycan expression of 27 organoid models. The classification of positivity at the bottom used cutoffs that optimized the discrimination between background signal and true marker expression. The magnification was 20X for the non-zoomed images.

Figure 2. Differential gene expression. A) Differentially expressed genes and pathways. B) Enrichment of previously identified sets in the sTRA-expressing cells. C) Relationships between class markers, morphology, and glycan expression across the cell lines. CYP3A5 and HNF1-a were significantly elevated in the sTRA group compared with the non-sTRA group after multipletesting correction D) KRAS mutation status associated with the glycan-defined groups. NMF, nonnegative matrix factorization to identify $C 1$ and $C 2$ clusters in the organoids. The $C / B$ column indicates the classical or basal status based on a gene set developed for the organoids.

Figure 3. Drug resistance differences between glycan-defined types. A) Single-dose viability in the panel of 27 cell lines. B) $I_{50}$ values calculated from dose-response curves, grouped by marker group. P Values were calculated by Mann-Whitney test. C) Summarized $\mathrm{IC}_{50}$ values for all drugs and cell lines. Z-scores were used to normalize the scales of the $\mathrm{IC}_{50}$ values for comparisons. D) Factors and markers associated with resistance. Z-scores were used to normalize the gene-expression data and the doubling times. Avg. Rank is the average across drugs of the ranks in $\mathrm{IC}_{50}$ of each cell line among the panel of 27.

Figure 4. A gene-expression classifier associated with drug resistance. A) Classification of patients using a signature for sTRA. The 28-gene classifier applied to the TCGA (top) and ICGC (bottom) datasets produced two distinct groups of patients. The color bar at top shows the classification used in the subsequent analyses. B) Survival curves grouped by the geneexpression classifier for sTRA positive or negative. C) Survival curves grouped by classical or basal status. The classical and basal classes were from a previous publication for the TCGA dataset and were calculated from the data for the ICGC dataset. The $p$ values are based on the log-rank test.

Figure 5. Tissue expression and outcomes. A) Raw immunofluorescence and detected signal of STRA and CA19-9 in representative cores from the TMAs. Magnification is 4X. B) Comparison between the CA19-9 and STRA levels in the patient tumors. Each point is the 


\section{Detecting Drug-Resistant PDAC}

average of the three cores for a patient. C) Comparisons of sTRA and CA19-9 immunofluorescence data between the patient groups stratified by survival. The dashed lines indicate the median values that were used as cutoffs in panel E. D) Receiver-operator characteristic analysis for each marker to distinguish short survival (the cases) from long survival (the controls). AUC, area under the curve. E) Response to adjuvant therapy in subgroups. Adj. indicates the patients who received adjuvant chemotherapy following surgery. Using the median values of either marker given in panel $C$, the patients were further stratified either by sTRA (left) or CA19-9 (right).

Figure 6. A blood test to predict rapid relapse following chemotherapy. A) Immunoassays used for measuring sTRA in patient plasma. B) Comparisons between short and long TTP, based on 18 months, of the indicated immunoassays. The values are the averages of three independent experiments. C) Patterns of high and low values across the three sTRA immunoassays in the discovery set. Each column represents a patient sample. Samples with elevation in two or three markers were classified as cases. D) Patterns of high and low values across the three sTRA immunoassays in the test set, using optimized thresholds. E) Survival curves in the discovery and test sets. For CA19-9, patients above the median were classified as high. For the panel, patients with elevations in two or three markers were classified as positive. 
Detecting Drug-Resistant PDAC

\section{Tables}

Table 1. Performance of the sTRA Panel and CA19-9 in the discovery and test sets. Cl, confidence interval. Bolded text of numbers indicates statistical significance. ${ }^{* *} p<0.0001,{ }^{*} p<$ 0.01 (Wald test with bootstrap standard error estimate).

\begin{tabular}{|c|c|c|c|c|}
\hline Sample set/performance & Marker & Sensitivity (95\% Cl) & Specificity $(95 \% \mathrm{Cl})$ & $\begin{array}{c}(\text { Sens + Spec }) / 2 \\
(95 \% \mathrm{Cl})\end{array}$ \\
\hline \multicolumn{5}{|l|}{ Discovery Set } \\
\hline \multirow[t]{2}{*}{ Naïve Performance } & sTRA Panel & 45.7 & 97.6 & 71.7 \\
\hline & CA19-9 & 2.9 & 95.2 & 49 \\
\hline \multirow[t]{3}{*}{$\begin{array}{l}\text { Cross-Validated } \\
\text { Performance }\end{array}$} & sTRA Panel & $45.4(28.2,63.7)$ & $92.5(80.7,97.3)$ & $68.9(57.7,78.3)$ \\
\hline & CA19-9 & $3.0(0.1,48.9)$ & $96.2(85.9,99.1)$ & $49.6(44.8,54.4)$ \\
\hline & Difference & **42.3 $(21.7,62.9)$ & $-3.7(-11.8,4.4)$ & ${ }^{* *} 19.3(8.1,30.6)$ \\
\hline \multicolumn{5}{|l|}{ Test Set } \\
\hline \multirow[t]{3}{*}{ Blinded Performance } & sTRA Panel & $55.6(37.2,72.5)$ & $96.4(78.1,99.5)$ & $76.0(64.8,84.5)$ \\
\hline & CA19-9 & $11.1(3.6,29.2)$ & $96.4(78.8,99.5)$ & $53.8(46.9,84.5)$ \\
\hline & Difference & ${ }^{* \star 44.4}(25.8,63.1)$ & $0(-10.1,10.1)$ & **22.2 $(11.6,32.9)$ \\
\hline \multirow[t]{2}{*}{ Naïve Performance } & sTRA Panel & 63 & 96.4 & 79.7 \\
\hline & CA19-9 & 11.1 & 96.4 & 53.8 \\
\hline \multirow[t]{3}{*}{$\begin{array}{l}\text { Cross-Validated } \\
\text { Performance }\end{array}$} & sTRA Panel & $66.2(44.8,82.5)$ & $90.6(75.4,96.8)$ & $78.4(64.6,87.8)$ \\
\hline & CA19-9 & $13.3(1.4,61.6)$ & $96.0(82.4,99.2)$ & $54.6(41.7,67.0)$ \\
\hline & Difference & **52.9 $(24.4,81.4)$ & $-5.4(-16.1,5.3)$ & *23.8 $(8.5,39.0)$ \\
\hline
\end{tabular}



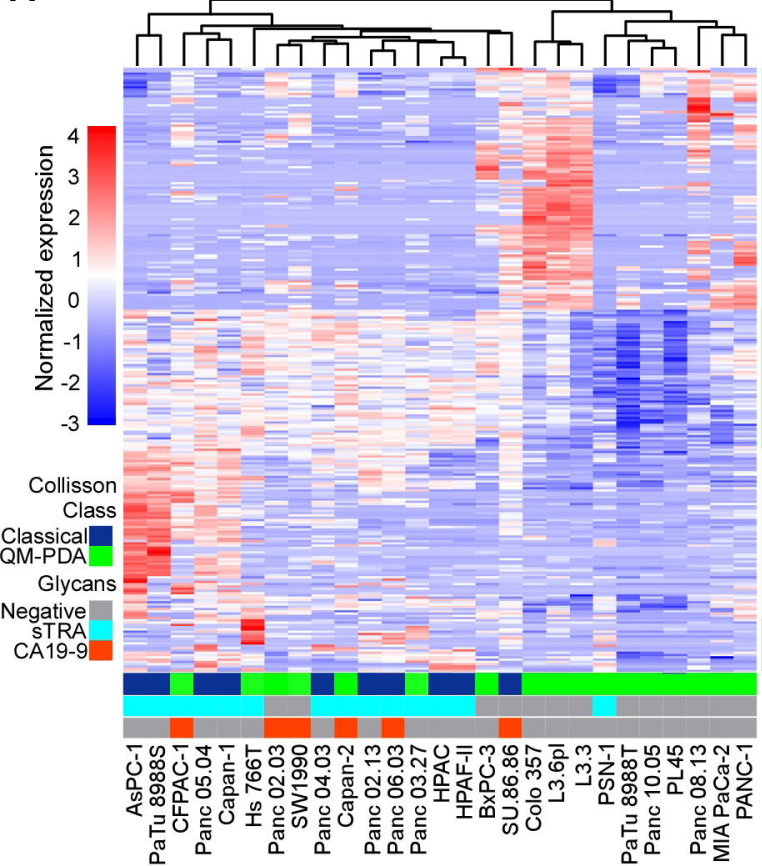

C

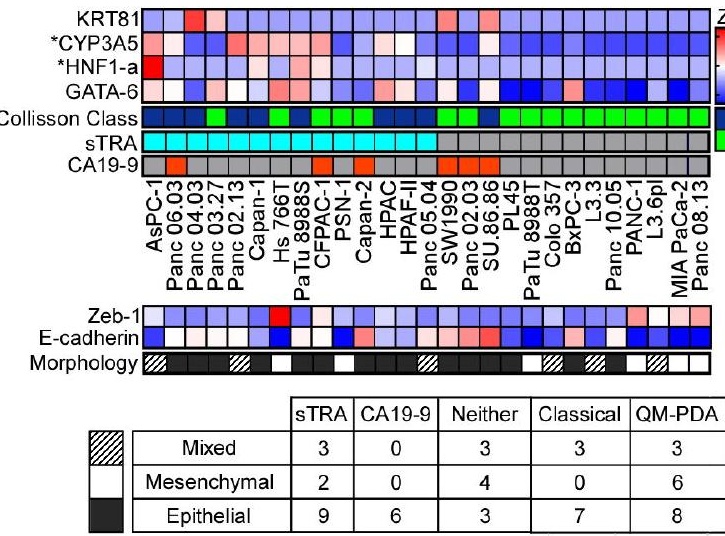

$\frac{\overline{0}}{0}$

B cell apoptotic process: BCL6; IRS2; BCL10 N Smooth muscle development: BMP4; PROX1 Bile acid biosynthesis: PROX1; NR1D1

Dorsal/Nentral axis specification: FZD5; SMAD6 Nucleotide-sugar metabolic: UGDH; GMDS Pancreas development: TCF7L2; PROX

을 Drug catabolic process: 은 CYP2B6; NR112; CYP3A5

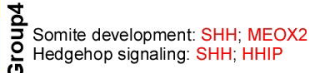

Red text: involved in development

贾造
Enrichment in sTRA-positive

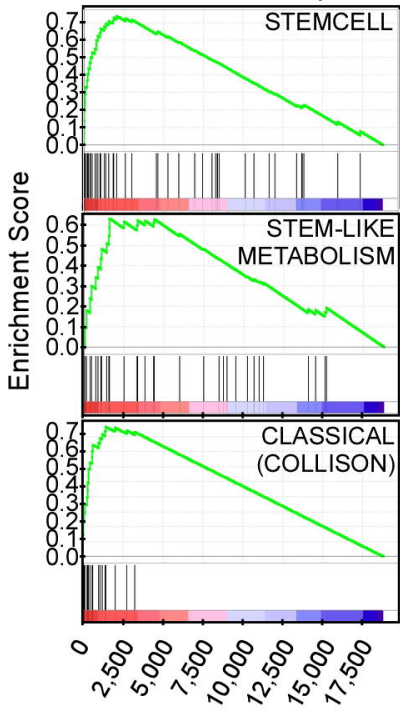

Rank in Ordered Dataset

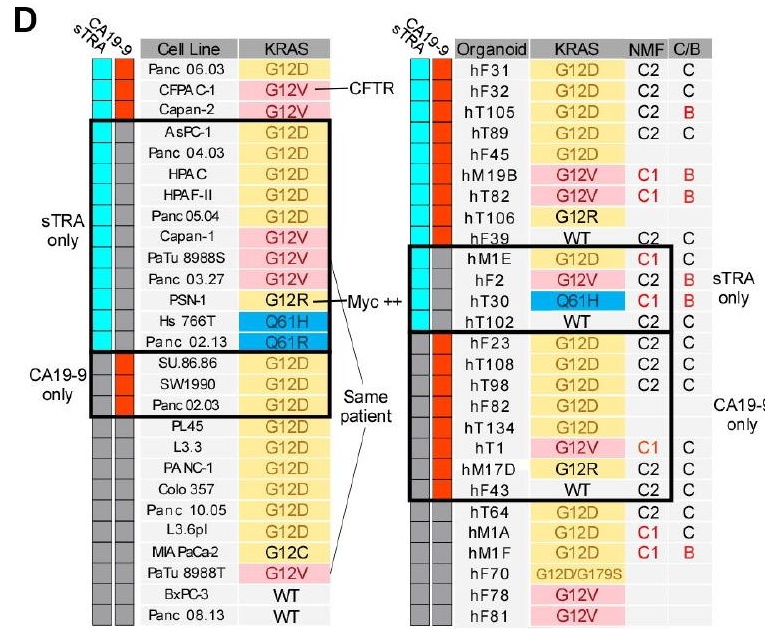




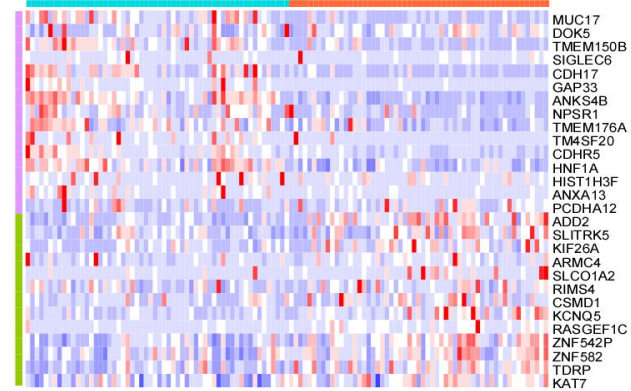

ICGC, $N=180$

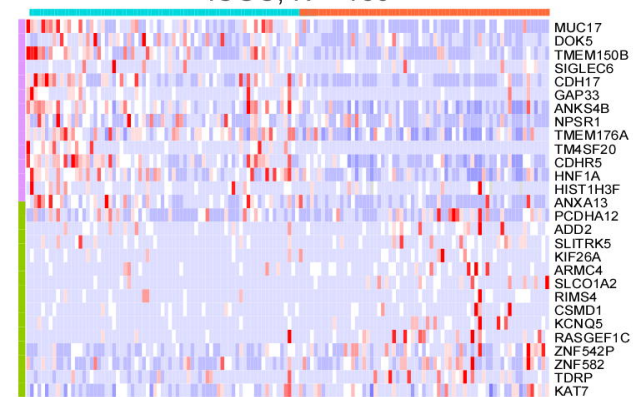
Patient classification
sTRA
Non-sTRA
Genes
Up-regulated
Down-regulated
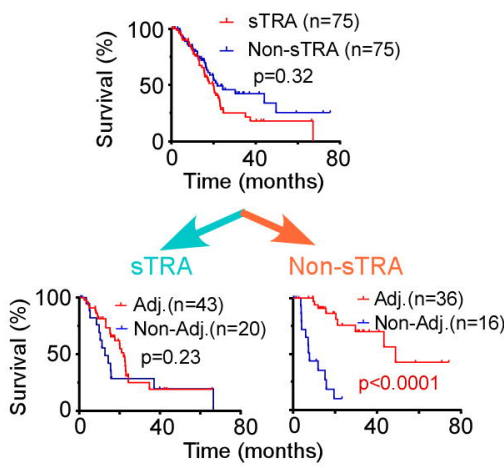

C

\section{TCGA}
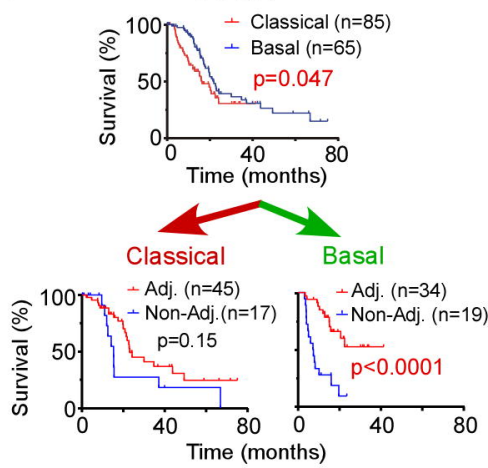
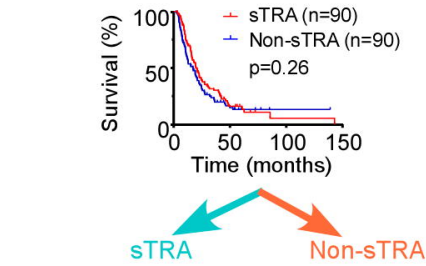

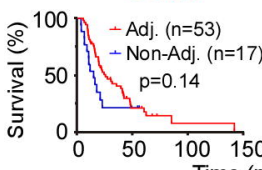

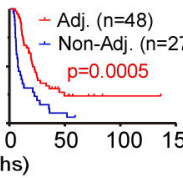

ICGC
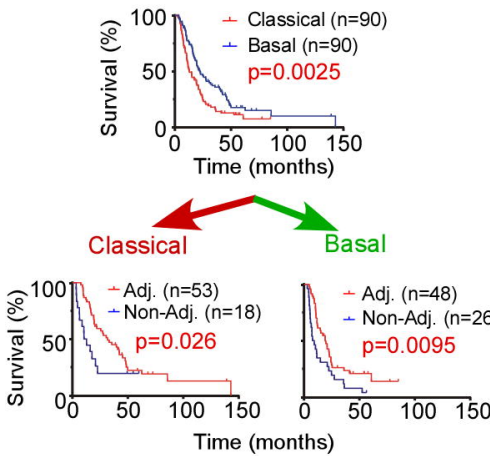
Time (months)

Relative expression 


\section{A $\quad$ Patient $47 \quad$ Patient $58 \quad$ Patient $64 \quad$ Patient 4}
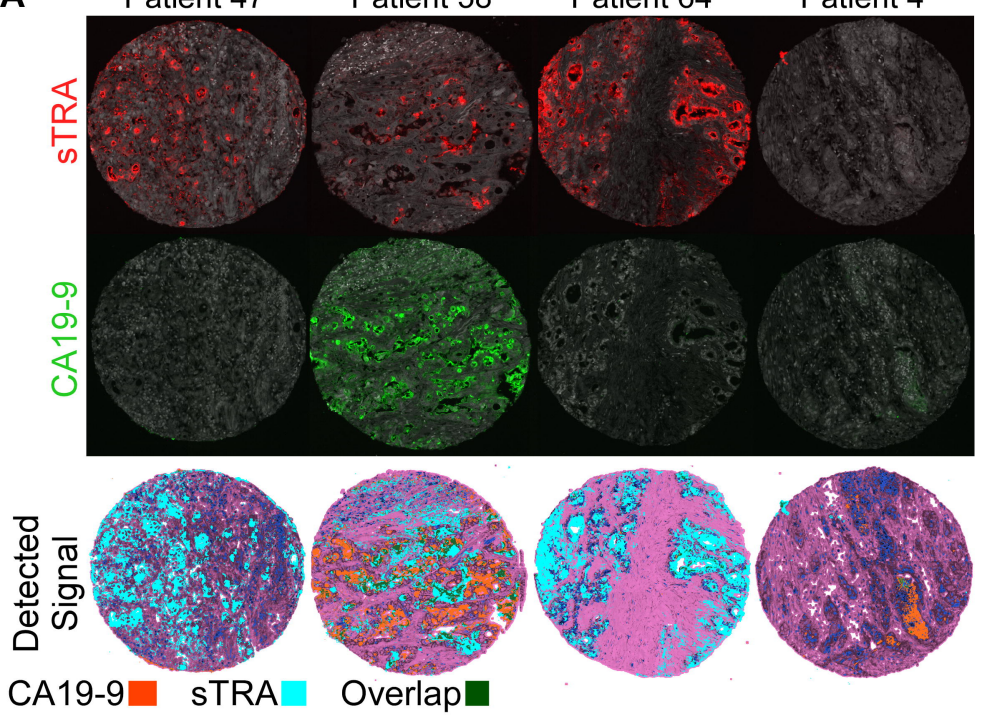

E

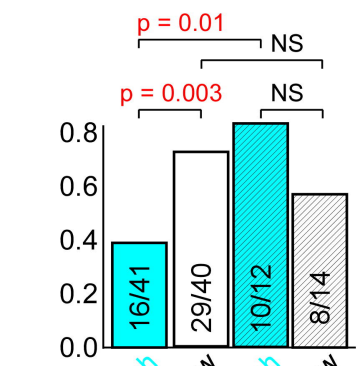

sTRA
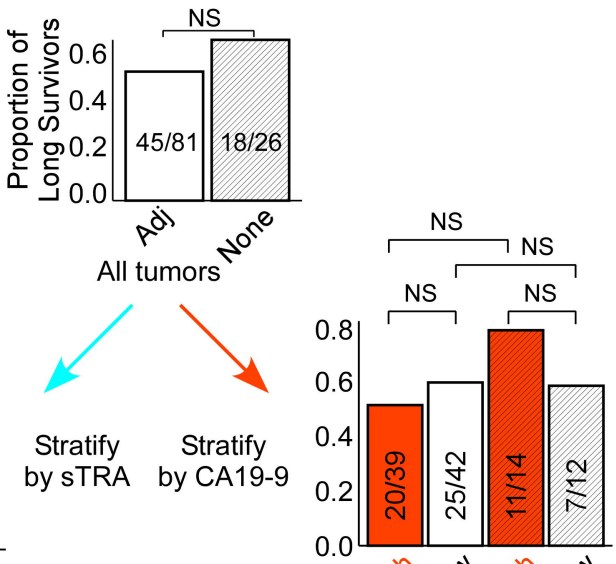

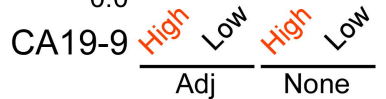

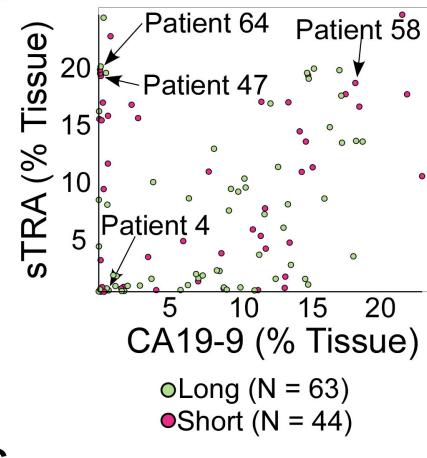

C

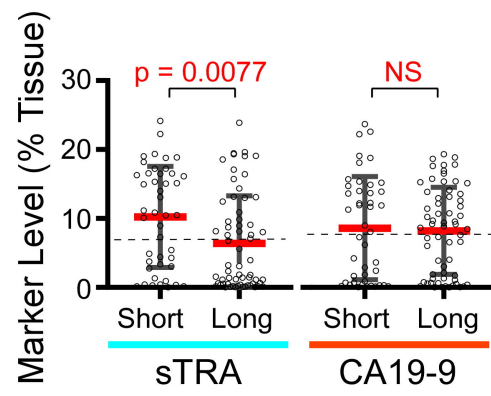

D

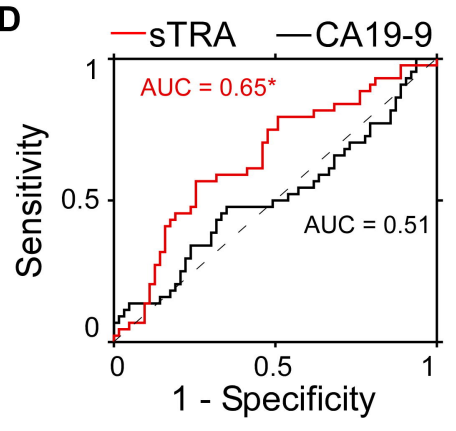




\section{A}
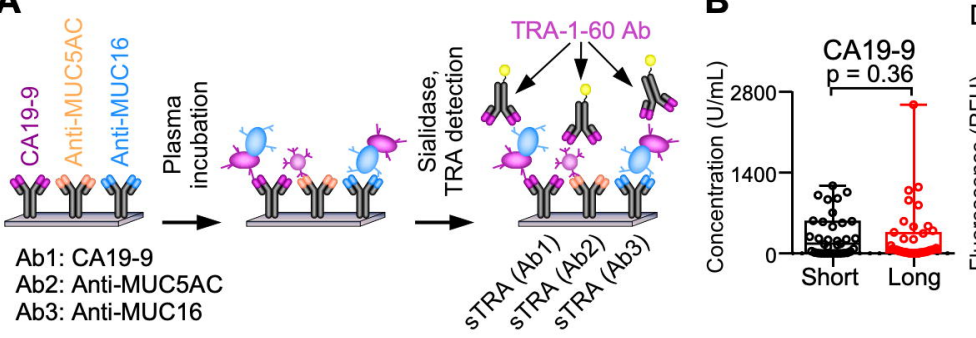

Ab3: Anti-MUC16

C

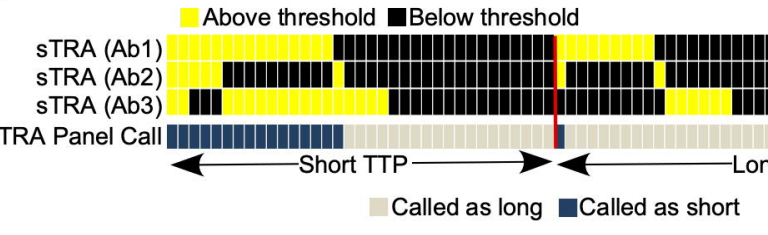

$98 \%$ Specificity
$46 \%$ Sensitivity

D

Test Set: $\mathrm{N}=27$ Short, 28 Long

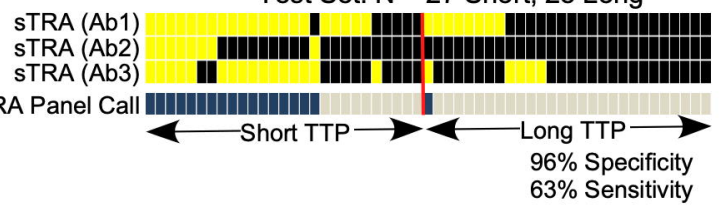

Discovery Set: $\mathrm{N}=35$ Short, 42 Long

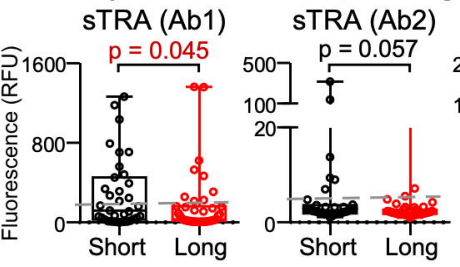

sTRA (Ab3)

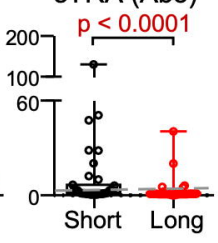

E
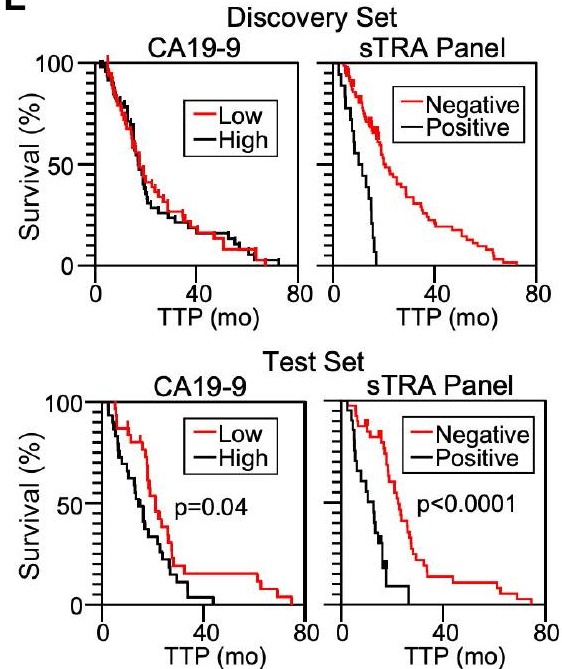